\title{
Resting-State Seed-Based Analysis: An Alternative to Task-Based Language fMRI and Its Laterality Index
}

\author{
(D) K.A. Smitha, D.M. Arun, DP.G. Rajesh, DB. Thomas, and (D) Kesavadas
}

\begin{abstract}
BACKGROUND AND PURPOSE: Language is a cardinal function that makes human unique. Preservation of language function poses a great challenge for surgeons during resection. The aim of the study was to assess the efficacy of resting-state fMRI in the lateralization of language function in healthy subjects to permit its further testing in patients who are unable to perform task-based fMRI.
\end{abstract}

MATERIALS AND METHODS: Eighteen healthy right-handed volunteers were prospectively evaluated with resting-state fMRI and taskbased fMRI to assess language networks. The laterality indices of Broca and Wernicke areas were calculated by using task-based fMRI via a voxel-value approach. We adopted seed-based resting-state fMRI connectivity analysis together with parameters such as amplitude of low-frequency fluctuation and fractional amplitude of low-frequency fluctuation (fALFF). Resting-state fMRI connectivity maps for language networks were obtained from Broca and Wernicke areas in both hemispheres. We performed correlation analysis between the laterality index and the $z$ scores of functional connectivity, amplitude of low-frequency fluctuation, and fALFF.

RESULTS: Pearson correlation analysis between signals obtained from the $z$ score of fALFF and the laterality index yielded a correlation coefficient of $0.849(P<.05)$. Regression analysis of the fALFF with the laterality index yielded an $R^{2}$ value of 0.721 , indicating that $72.1 \%$ of the variance in the laterality index of task-based fMRI could be predicted from the fALFF of resting-state fMRI.

CONCLUSIONS: The present study demonstrates that fALFF can be used as an alternative to task-based fMRI for assessing language laterality. There was a strong positive correlation between the fALFF of the Broca area of resting-state fMRI with the laterality index of task-based fMRI. Furthermore, we demonstrated the efficacy of fALFF for predicting the laterality of task-based fMRI.

ABBREVIATIONS: ALFF = amplitude of low-frequency fluctuation; BOLD = blood oxygen level-dependent; fALFF = fractional amplitude of low-frequency fluctuation; $\mathrm{FC}$ = functional connectivity; $\mathrm{LI}=$ laterality index; rsfMRI = resting-state fMRI

B rain surgery demands preservation of eloquent areas, including the language functional areas. Many studies have illustrated that $\mathrm{fMRI}$ is a noninvasive imaging technique that facilitates the lateralization of language function in individual patients. ${ }^{1-3}$ The hemispheric language laterality index (LI) is estimated by evaluating the asymmetry in the activation of language areas in the right and left hemispheres of the human brain during a particular task. ${ }^{4}$ The ease of integrating fMRI with other MR

Received June 25, 2016; accepted after revision February 2, 2017.

From the Departments of Imaging Sciences and Interventional Radiology (K.A.S K.M.A., B.T., C.K.) and Neurology (P.G.R.), Sree Chitra Tirunal Institute for Medical Sciences and Technology, Trivandrum, Kerala, India.

This work was supported by the Department of Biotechnology, Ministry of Science and Technology, Government of India.

Please address correspondence to K.A. Smitha, PhD, Department of Imaging Sciences and Interventional Radiology, Sree Chitra Tirunal Institute for Medical Sciences and Technology, Trivandrum, Kerala, India, Pin code: 695011; e-mail: mithamahesh@gmail.com

- Indicates open access to non-subscribers at www.ajnr.org

http://dx.doi.org/10.3174/ajnr.A5169 imaging techniques allows the correct identification of pathology and its landmark with respect to language areas. ${ }^{5-7}$

During the acquisition of language fMRI, the subject is instructed to perform particular tasks designed to elicit a response from language functional areas. However, not all subjects, such as those with low intelligence quotients and pediatric patients, will be able to cooperate with the task-based fMRI. Resting-state fMRI (rsfMRI) is gradually evolving as an alternative to task-based fMRI. The rsfMRI technique extracts the low-frequency fluctuations in the blood oxygen level-dependent (BOLD) signal when subjects are instructed to lie relaxed inside the scanner. ${ }^{7,8}$

Tie et $\mathrm{al}^{9}$ extracted language networks from rsfMRI and evaluated the effectiveness of the automatic identification of language components by using independent component analysis. The study separated the activation patterns corresponding to the language network components from individual rsfMRI data. Another study adopted rsfMRI connectivity analysis to examine the degree of hemispheric dominance for language processing in healthy controls and patients with temporal lobe epilepsy. ${ }^{10}$ 
Functional connectivity (FC) analysis of the frontal cortex in the control group revealed a strong correlation with the LI. The authors found a good correlation between the LI and the epileptogenic mesial temporal lobe. Similarly, Pravatà et $\mathrm{al}^{11}$ studied the correlation between the strength of FC between language networks and language performance in healthy controls and patients with epilepsy. They demonstrated an overall reorganization and reduction of the connectivity pattern within the language network of patients with intractable epilepsy. These studies evaluated the FC of language networks rather than regional abnormalities in the low-frequency fluctuations.

In rsfMRI, the parameters FC, amplitude of low-frequency fluctuation (ALFF), and fractional amplitude of low-frequency fluctuation (fALFF) are used to examine the network-related and regional characteristics of low-frequency oscillations. FC analysis evaluates the correlation between the time courses of voxels in a seed region with every other region within the brain. The regions with strong correlations will be shown as an FC map. ${ }^{7,12}$ ALFF and fALFF are rsfMRI metrics that help in identifying regional BOLD signal changes of rsfMRI fluctuations. ALFF quantifies the amplitude of the low-frequency fluctuations of rsfMRI BOLD signals. fALFF corresponds to the power spectrum of the low-frequency band with respect to all frequencies detectable with the applied time of repetition of the study. ${ }^{13,14}$ Different studies reported the clinical application of these metrics. ${ }^{13,15,16}$ However, none of the studies considered the accuracy of these metrics in determining the LI. ${ }^{9-11}$

The objective of our study was to determine the potential of rsfMRI to evaluate language lateralization in comparison with task-based fMRI. In the current study, we evaluated the $z$ scores of FC, ALFF, and fALFF obtained via rsfMRI and assessed the correlations of each of these metrics with the LI of task-based fMRI from individual seed regions. We hypothesized that the metrics of seed-based rsfMRI analysis are strongly correlated with the LI obtained by using task-based fMRI. If this hypothesis is proved, then rsfMRI can be used instead of task-fMRI in patients who cannot perform the task.

\section{MATERIALS AND METHODS}

In this prospective observational study, MR imaging was performed in 18 healthy right-handed volunteers (12 men and 6 women). The Edinburgh Handedness Inventory was used to evaluate the handedness of the subjects. The mean age of the cohort was $30 \pm 7.8$ years. Subject scanning was conducted by using a 1.5T MR imaging scanner (Magnetom Avanto, Tim; Siemens, Erlangen, Germany). Informed consent was obtained from all participating subjects. The study received clearance from the institutional ethics committee. The imaging sequence included structural imaging, rsfMRI, and task-based fMRI. Structural imaging was performed by using the MPRAGE sequence with the following imaging protocol: $\mathrm{TR}=2400 \mathrm{~ms}, \mathrm{TE}=3.72 \mathrm{~ms}, \mathrm{TI}=$ $1000 \mathrm{~ms}, \mathrm{NEX}=2$, flip angle $=8^{\circ}$, section thickness $=1 \mathrm{~mm}$, imaging matrix $=256 \times 256$ with an isotropic voxel of $1 \times 1 \mathrm{~mm}$. Resting-state fMRI was acquired with a gradient-echo EPI sequence with the following imaging protocol: $\mathrm{TR}=2000 \mathrm{~ms}, \mathrm{TE}=$ $20 \mathrm{~ms}$, flip angle $=90^{\circ}$, section thickness $=5 \mathrm{~mm}$, number of sections $=25$ acquired in the interleaved bottom up order, imag- ing matrix $=320 \times 320$ with an isotropic voxel of $3.75 \times 3.75$. During rsfMRI, subjects were instructed to concentrate on the white crosshair on the black background displayed on the screen.

Subsequently, task-based fMRI was performed with the same imaging protocol. Language fMRI was conducted with the visualverb paradigm. ${ }^{17}$ During the active block, subjects were instructed to think about the verb associated with the pictures presented on the screen. During the rest block, subjects were instructed to concentrate on the white crosshair on the black background displayed on the screen. The stimulus presentation paradigm was designed as blocks of tasks and rest with 5 cycles ( 5 rest and 5 active blocks).

\section{Image Analysis}

The reconstructed DICOM images were converted to the NIfTI format for further processing. We used SPM8 (http://www.fil.ion. ucl.ac.uk/spm/software/spm12) to process task-based fMRI. The rsfMRI processing was performed by using the Data Processing Assistant for Resting State fMRI (http://restfmri.net/forum/ DPARSF), the Resting-State fMRI Data Analysis Toolkit (REST; http://www.restfmri.net), and RESTplus software (http://restfmri. net/forum/RESTplusV1.2). ${ }^{18,19}$

The NIfTI images were preprocessed in the SPM pipeline. The images were realigned, section time-corrected, and normalized. The normalized images were smoothed with a Gaussian kernel at a full width at half maximum of $8 \mathrm{~mm}$. The general linear model design matrix was defined with a canonical hemodynamic response function as the basis function, and it was estimated to obtain $\beta$ values. Furthermore, we performed family-wise error correction for individual subjects $(P<.05)$. Six ROIs from both the left and right hemispheres of the Broca area, Brodmann area 6 , and Wernicke area were identified. Using MarsBaR software (https://sourceforge.net/projects/marsbar/files/), we created spheric masks with Montreal Neurological Institute coordinates to obtain these ROIs. ${ }^{20}$ These masks were used to derive the LI by using the LI Toolbox (http://www.medizin.uni-tuebingen.de/ kinder/en/research/neuroimaging/software/) implemented in SPM8. We used a method called "voxel value of LI" to calculate the activated voxels, in which the value of the voxel represents the strength of its correlation with the task. ${ }^{21}$ The LI varies from -1 to +1 . An individual with a positive LI is said to be left-lateralized, and a person with a negative LI is right-lateralized. The equation for LI is as follows $^{4,17,22,23}$ :

$$
L I=\Sigma L i-\Sigma R i / \Sigma L i+\Sigma R i
$$

where $\Sigma L i$ is the number of voxels activated at a particular threshold in the left hemisphere of the ROI and $\Sigma R i$ is that of the right hemisphere.

For rsfMRI, the same pipeline of preprocessing used for taskbased fMRI was followed. Nuisance covariate regression and temporal bandpass filtering in the range of $0.01-0.08 \mathrm{~Hz}$ were performed for FC analysis. The Brodmann areas 47, 45, 44, 6, 21, and 22 of both hemispheres were taken as seed regions for rsfMRI analysis. After performing seed-based FC analysis, ALFF and fALFF were calculated by using RESTplus software. 


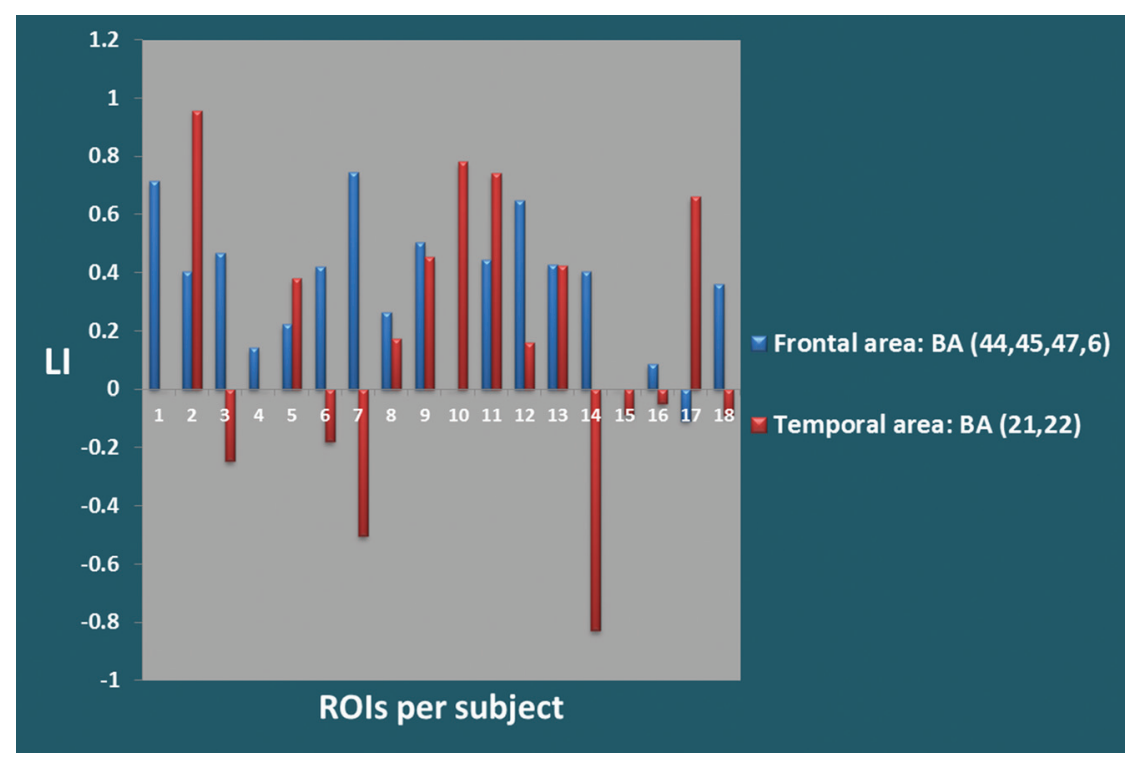

FIG 1. The bar chart presents the average value of the laterality indices obtained by using task-based fMRI for all the subjects in the Broca area, Brodmann area 6 (frontal area), and Wernicke area (temporal area).

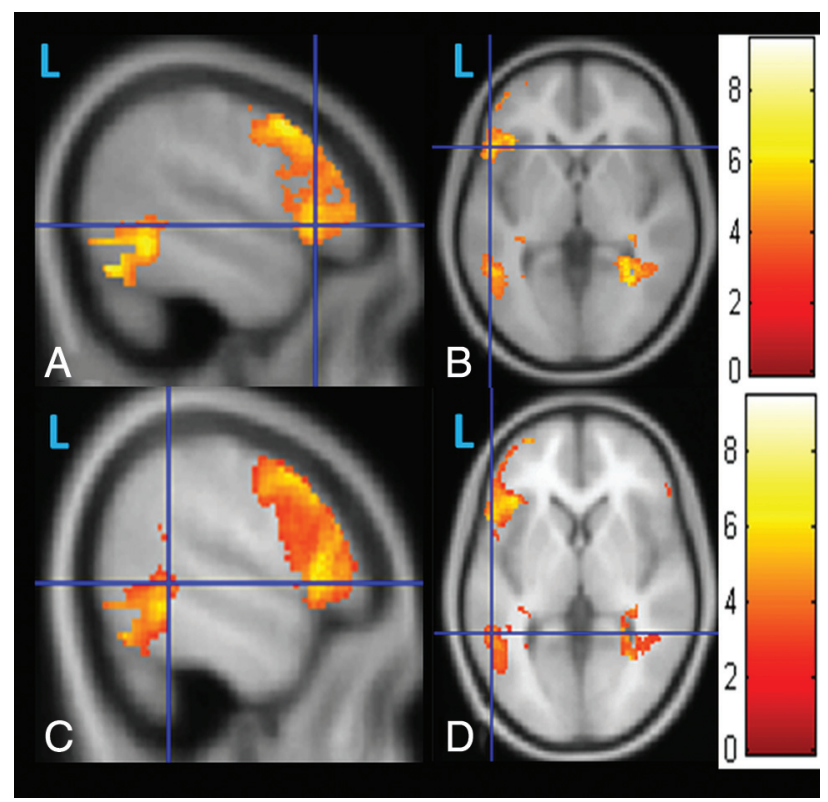

FIG 2. $A$ and $B$, Sagittal and axial images obtained after the 1-sample $t$ test of the task-fMRI for the Broca area and Brodmann area 6. $C$ and $D$, Wernicke area of 18 healthy controls.

\section{Statistical Analysis}

Statistical analysis was performed by using Excel (Microsoft, Redmond, Washington) and SPSS, Version 17 for Windows (IBM, Armonk, New York). The FC, ALFF, and fALFF scores were converted into $z$ scores by using the Fisher transformation. A 1-sample $t$ test on $Z$-maps of FC, ALFF, and fALFF on all subjects yielded a $T$-map. This was performed by using RESTplus software. Thereafter, the FC, ALFF, and fALFF values of the corresponding ROIs were extracted from the T-map and correlated with the LI.

Pearson correlation analysis was conducted to find the associations between rsfMRI metrics and the LI. Linear regression analysis and $R^{2}$ values were obtained only for the specific rsfMRI metrics that showed a significant correlation with LI.

\section{RESULTS}

LI values were computed from all individual ROIs. Figure 1 presents the bar diagram of LI for each of the ROIs from all subjects. Figure 2 indicates the task-based fMRI BOLD signal activations obtained from the Broca area, Brodmann area 6, and Wernicke area.

The seed-based rsfMRI connectivity analysis was performed in the language regions of both hemispheres, such as the Broca area, Brodmann area 6, and Wernicke area. The $Z$-maps corresponding to FC, ALFF, and fALFF for the Broca area are depicted in Fig 3. Table 1 shows the mean value of the LI obtained for all subjects for the respective ROIs. Table 2 presents the Pearson correlation coefficient obtained between the rsfMRI seedbased metrics for the respective ROIs with the LI obtained from task-based fMRI. When $z$ scores extracted from the Broca area were analyzed, only fALFF displayed a statistically significant correlation with the LI with a correlation coefficient of $0.849(P<.032)$. Regression analysis of the $z$ score obtained from fALFF analysis and the LI yielded an $R^{2}$ value of 0.721 , as shown in Fig 4. Similarly, a positive correlation coefficient of 0.531 was observed between the $z$ score of FC and the LI, and a negative correlation coefficient of -0.153 was obtained between the $z$ score of ALFF and LI. When $z$ scores extracted from the Wernicke area and Brodmann area 6 were analyzed, we observed a weak positive correlation between the $z$ scores of ALFF and fALFF with the LI, whereas a negative correlation was observed between FC and the LI, as shown in Table 2.

\section{DISCUSSION}

The current study evaluated the effectiveness of rsfMRI in predicting the language dominance of hemispheres in healthy controls by correlating the rsfMRI metrics FC, ALFF, and fALFF with the LI of task-based fMRI. We studied task-based fMRI activation in the Broca area, Wernicke area, and Brodmann area 6 and compared the findings with those of rsfMRI connectivity analysis in the same areas as the seed regions. We evaluated the $z$ scores of FC, ALFF, and fALFF of the respective areas for all subjects and correlated the values with the LI of task-based fMRI. These connectivity metrics have been analyzed to focus on the frequency-specific characteristics of rsfMRI networks. ${ }^{24}$ ALFF is the average amplitude of the low-frequency band $(0.08-0.1 \mathrm{~Hz})$ because it measures the magnitude of spontaneous neuronal activity. ${ }^{14,25}$ fALFF is the ratio between the power spectrum of the low-frequency signal and the entire frequency range that is dependent on the TR of the imaging protocol. Consequently, the peculiarity of fALFF with respect to ALFF is that it markedly reduces the noises in the highfrequency band in cisternal regions of the brain. ${ }^{13-15,26-29}$

Numerous studies have investigated the clinical applications of ALFF and fALFF metrics. ${ }^{13-16,27-29}$ Turner et $\mathrm{al}^{13}$ examined ALFF and fALFF to clarify differences between patients with 


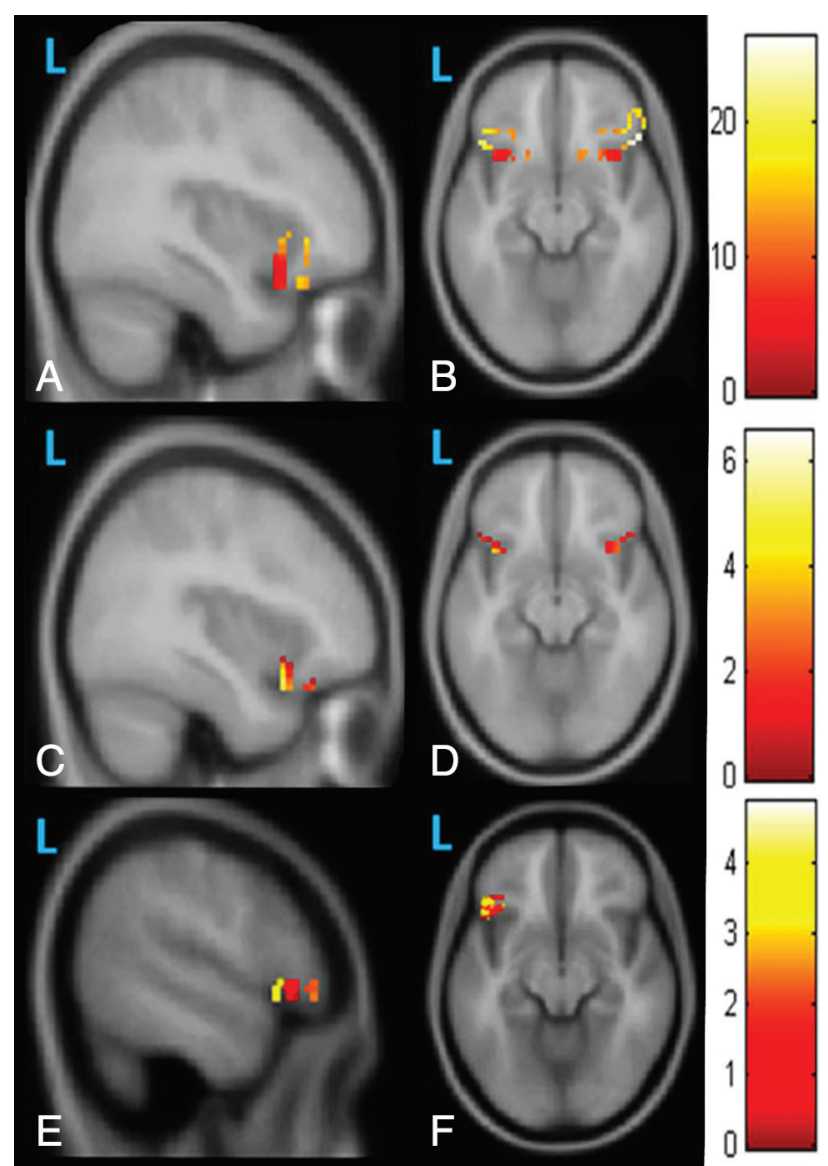

FIG 3. The first row shows the sagittal and axial images obtained after a 1-sample $t$ test of the Z-maps of 18 functional connectivity images obtained with the Broca area as the seed area. The second row shows sagittal and axial images obtained after a l-sample $t$ test of the $Z$-maps of the amplitude of low-frequency fluctuations in 18 healthy controls. We observed limited correlated activity for functional connectivity ( $A$ and $B$ ) and ALFF ( $C$ and $D$ ); it seems to be fragmented and potentially less clinically reliable at the individual level. The third row shows the sagittal and axial images obtained after a 1-sample $t$ test of the Z-maps of fractional amplitude of low-frequency fluctuations in 18 healthy controls. By contrast, the map of fALFF ( $E$ and $F$ ) appears more robust and potentially more reliable.

Table 1: The mean value of the laterality index obtained from Brodmann areas 44, 45, 47, 6, 21, and 22 using task-based fMRI for all subjects

\begin{tabular}{lc}
\hline \multicolumn{1}{c}{ Language Areas } & Mean LI of 18 Subjects \\
\hline Brodmann Area 44 & 0.375 \\
Brodmann Area 45 & 0.238 \\
Brodmann Area 47 & 0.430 \\
Brodmann Area 6 & 0.377 \\
Brodmann Area 21 & 0.166 \\
Brodmann Area 22 & 0.218 \\
\hline
\end{tabular}

schizophrenia and healthy controls. The study analyzed these metrics from different sites and found that patients with schizophrenia had a lower fALFF than healthy controls across the cortex. Another group found variations in ALFF and fALFF associated with the right precuneus and left medial prefrontal gyrus and suggested that the changes in these measures can be used as surrogate markers of minimal hepatic encephalopathy. ${ }^{16}$

In our study, the $z$ scores of FC, ALFF, and fALFF were extracted from Broca and Wernicke areas separately. The $Z$-map of
Table 2: Correlation of the seed-based analysis metrics FC, ALFF, and fALFF of rsfMRI with the LI of task-based fMRI

\begin{tabular}{lccc}
\hline & $\begin{array}{c}\text { Correlation } \\
\text { between the } \\
\text { Z Score of } \\
\text { Region }\end{array}$ & $\begin{array}{c}\text { Correlation } \\
\text { between the } \\
\text { Z Score of }\end{array}$ & $\begin{array}{c}\text { Correlation } \\
\text { between the } \\
\text { Z Score of } \\
\text { fALFF and LI }\end{array}$ \\
\hline $\begin{array}{l}\text { Broca area } \\
\text { Wernicke area } \\
\text { and Brodmann } \\
\text { area 6 }\end{array}$ & $\begin{array}{c}0.531(P=.277) \\
-0.752(P=.085)\end{array}$ & $\begin{array}{c}-0.153(P=.771) \\
0.182(P=.729)\end{array}$ & $0.379(P=.032)$ \\
\hline
\end{tabular}

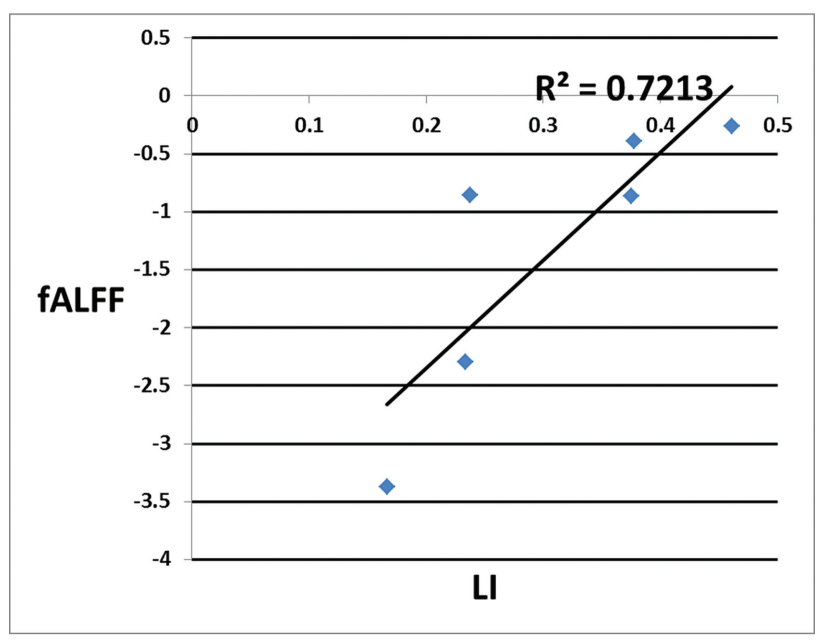

FIG 4. The regression line plotted with signals extracted from the $T$-map of the $z$ scores of the fractional amplitude of low-frequency fluctuations from the Broca area of resting-state fMRI with the laterality index obtained from all ROls of task-based fMRI.

fALFF obtained from rsfMRI seed-based analysis revealed that the Broca area is mainly left hemisphere-lateralized. In addition, we observed a strong correlation between the $z$ score of fALFF obtained from the Broca area on rsfMRI and the LI obtained from task-based fMRI. Regression analysis of the $z$ score of fALFF and LI showed a noticeable $R^{2}$ value, indicating the usefulness of rsfMRI connectivity analysis in predicting the LI of language areas. Meanwhile, a weak positive correlation coefficient of 0.372 was observed when the $z$ score of fALFF in Brodmann area 6 and the Wernicke area was correlated with the LI of task-based fMRI.

To the best of our knowledge, including a review of the literature, no previous study evaluated rsfMRI FC analysis and taskbased fMRI to correlate resting-state connectivity metrics with LIs associated with task-based fMRI. From the individual cases of task-based fMRI, we observed that BOLD activations in the Broca area in most subjects were left-lateralized; conversely, for the Wernicke area, half of the subjects were right-lateralized or they had an LI close to zero, as shown in Fig 1. We therefore consider this finding is a reason for the negative correlation between the $z$ score of FC of the Wernicke area and the LIs of the respective ROIs. Thus, the task-based fMRI analysis demonstrated more consistency in Broca activation and more variability in Wernicke activation. Our findings reveal that frontal lateralization is more robust than temporal activation. This result may be because frontal activity is more easily detected than temporal activity, which can be masked by susceptibility artifacts. ${ }^{17}$ Zhu et al ${ }^{30}$ examined rsfMRI seed-based FC with Broca and Wernicke regions as seed regions. It is evident from their observations that the activations in the Broca 
area were left-lateralized, and those in the Wernicke area remained right-lateralized. The outcome of their analysis is in line with our results from task-based fMRI. Their study did not estimate the seed-based rsfMRI metrics; instead, they assessed the consistency of rsfMRI language networks and found that language networks are highly reproducible.

Doucet et $\mathrm{al}^{10}$ provided evidence that resting-state FC can be used to predict the strength of hemispheric language laterality in patients with temporal lobe epilepsy and controls. The ability of regional resting-state FCs to predict the LI was determined and compared. Although the results of the present study are in line with those of Doucet et al, additional metrics for predicting LI such as ALFF and fALFF were investigated. ${ }^{10}$ We hypothesized that FC analysis alone may not be sufficient to uncover subtle abnormalities in low-frequency fluctuations of BOLD signals and that the use of ALFF and fALFF can disclose more meaningful information. Although ALFF simply measures the power of the low-frequency fluctuation of a specific ROI, the negative correlation between ALFF in the Broca area and the LI may be due to the lower power of filtered frequency signals within the low-frequency band $(0.01-0.08 \mathrm{~Hz})$ in the Broca area in healthy subjects during the resting-state than during a task. ${ }^{13,14,16,31}$ Zou et al ${ }^{14}$ observed that fALFF exhibits greater sensitivity and specificity than ALFF.

Pravatà et $\mathrm{al}^{11}$ investigated the correlation between $\mathrm{FC}$ and neuropsychological evaluations of language. The study observed an overall decrease in FC within the language network of patients with intractable epilepsy compared with that in controls. ${ }^{11}$ The study did not consider the efficacy of FC to calculate language lateralization, but it emphasized that FC analysis provides a more illustrative assessment of functional modification. Tie et $\mathrm{al}^{9}$ investigated the feasibility of rsfMRI in right-handed healthy controls by using the group independent component analysis method. A new semiautomated method was used to identify the language components and compare rsfMRI and task-based fMRI activations in the language area. Rather than the LI, they adopted the Dice coefficient to determine the overlap between the language network areas from rsfMRI and task-based fMRI. The group-level analysis of task-based fMRI and rsfMRI uncovered markedly similar language regions in right-handed subjects. Resting-state fMRI identified more left lateralization and suggested that the semiautomatic language component identification procedure provides the best strategy for rsfMRI with the independent component analysis technique.

A study with a larger number of subjects, including more right-language-lateralized subjects, and thinner sections and a higher Tesla MR imaging device is required to confirm our findings. In addition, we need to investigate whether we can reproduce these results in a patient cohort by validating the results with neuropsychology analysis and the Wada test for language lateralization. Methodologic improvements such as automatic parcellation of language regions may strengthen the reliability of the analysis in the future. This method of analysis can provide better results than the Montreal Neurological Institute-based spheric ROI method. A cross-validation with other rsfMRI analysis techniques would also be useful.

\section{CONCLUSIONS}

The current study highlighted a strong correlation between the seed-based rsfMRI metric fALFF of the Broca area and the LI of task-based fMRI. The study demonstrated that rsfMRI connectivity analysis can be used for assessing language networks and fALFF can be used as an effective predictor for evaluating language laterality. The outcome of the study might be useful in testing the efficacy of rsfMRI in patients who are unable to perform taskbased $\mathrm{fMRI}$ in preoperative language lateralization.

\section{ACKNOWLEDGMENTS}

We acknowledge Professor Sankara Sarma for his valuable advice regarding the statistical analysis methods.

Disclosures: K.A. Smitha—RELATED: Grant: Research Associateship, Department of Biotechnology, Ministry of Science and Technology, Government of India. Bejoy Thomas-UNRELATED: Grants/Grants Pending: GE Healthcare, Comments: research project grant*; Patents (Planned, Pending or Issued): Sree Chitra Tirunal Institute for Medical Sciences and Technology (employer), Comments: Indian patent applied, unrelated to the current publication*. Chandrasekharan KesavadasRELATED: Grant: research funded by Department of Biotechnology, Ministry of Science and Technology, Government of India*; UNRELATED: Grants/Grants Pending: GE Healthcare*. *Money paid to the institution.

\section{REFERENCES}

1. Ruff IM, Petrovich Brennan NM, Peck KK, et al. Assessment of the language laterality index in patients with brain tumor using functional MR imaging: effects of thresholding, task selection, and prior surgery. AJNR Am J Neuroradiol 2008;29:528 -35 CrossRef Medline

2. Zaca D, Jarso S, Pillai JJ. Role of semantic paradigms for optimization of language mapping in clinical fMRI studies. AJNR Am J Neuroradiol 2013;34:1966-71 CrossRef Medline

3. James JS, Kumari SR, Sreedharan RM, et al. Analyzing functional, structural, and anatomical correlation of hemispheric language lateralization in healthy subjects using functional MRI, diffusion tensor imaging, and voxel-based morphometry. Neurol India 2015;63: 49-57 CrossRef Medline

4. Seghier ML. Laterality index in functional MRI: methodological issues. Magn Reson Imaging 2008;26:594-601 CrossRef Medline

5. Garrett MC, Pouratian N, Liau LM. Use of language mapping to aid in resection of gliomas in eloquent brain regions. Neurosurg Clin $N$ Am 2012;23:497-506 CrossRef Medline

6. Gupta S. fMRI for mapping language networks in neurosurgical cases. Indian J Radiol Imaging 2014;24:37-43 CrossRef Medline

7. Biswal BB. Resting state fMRI: a personal history. Neuroimage 2012; 62:938-44 CrossRef Medline

8. Lee MH, Smyser CD, Shimony JS. Resting-state fMRI: a review of methods and clinical applications. AJNR Am J Neuroradiol 2013;34: 1866-72 CrossRef Medline

9. Tie $\mathrm{Y}$, Rigolo L, Norton IH, et al. Defining language networks from resting-state fMRI for surgical planning: feasibility study. Hum Brain Mapp 2014;35:1018-30 CrossRef Medline

10. Doucet GE, Pustina D, Skidmore C, et al. Resting-state functional connectivity predicts the strength of hemispheric lateralization for language processing in temporal lobe epilepsy and normals. Hum Brain Mapp 2015;36:288-303 CrossRef Medline

11. Pravatà E, Sestieri C, Mantini D, et al. Functional connectivity MR imaging of the language network in patients with drug-resistant epilepsy. AJNR Am J Neuroradiol 2011;32:532-40 CrossRef Medline

12. Biswal B, Yetkin FZ, Haughton VM, et al. Functional connectivity in the motor cortex of resting human brain using echo-planar MRI. Magn Reson Med 1995;34:537-41 CrossRef Medline

13. Turner JA, Damaraju E, van Erp TG, et al. A multi-site resting state fMRI study on the amplitude of low frequency fluctuations in schizophrenia. Front Neurosci 2013;7:137 CrossRef Medline

14. Zou QH, Zhu CZ, Yang Y, et al. An improved approach to detection 
of amplitude of low-frequency fluctuation (ALFF) for resting-state fMRI: fractional ALFF. J Neurosci Methods 2008;172:137-41 CrossRef Medline

15. Tadayonnejad R, Yang S, Kumar A, et al. Clinical, cognitive, and functional connectivity correlations of resting-state intrinsic brain activity alterations in unmedicated depression. J Affect Disord 2015; 172:241-50 CrossRef Medline

16. Zhong WJ, Zhou ZM, Zhao JN, et al. Abnormal spontaneous brain activity in minimal hepatic encephalopathy: resting-state fMRI study. Diagn Interv Radiol 2016;22:196-200 CrossRef Medline

17. Rosazza C, Ghielmetti F, Minati L, et al. Preoperative language lateralization in temporal lobe epilepsy (TLE) predicts peri-ictal, preand post-operative language performance: an fMRI study. Neuroimage Clin 2013;3:73-83 CrossRef Medline

18. Chao-Gan Y, Yu-Feng Z. DPARSF: A MATLAB toolbox for "pipeline" data analysis of resting-state fMRI. Front Syst Neurosci 2010; 4:13 CrossRef Medline

19. Song XW, Dong ZY, Long XY, et al. REST: a toolkit for resting-state functional magnetic resonance imaging data processing. PLoS One 2011;6:e25031 CrossRef Medline

20. Wang L, Chen D, Yang X, et al. Group independent component analysis and functional MRI examination of changes in language areas associated with brain tumors at different locations. PLoS One 2013;8:e59657 CrossRef Medline

21. Wilke M, Lidzba K. LI-tool: a new toolbox to assess lateralization in functional MR-data. J Neurosci Methods 2007;163:128-36 CrossRef Medline

22. Binder JR, Swanson SJ, Hammeke TA, et al. Determination of lan- guage dominance using functional MRI: a comparison with the Wada test. Neurology 1996;46:978-84 CrossRef Medline

23. Desmond JE, Sum JM, Wagner AD, et al. Functional MRI measurement of language lateralization in Wada-tested patients. Brain 1995;118(pt 6):1411-19 CrossRef Medline

24. Zuo XN, Di Martino A, Kelly C, et al. The oscillating brain: complex and reliable. Neuroimage 2010;49:1432-45 CrossRef Medline

25. Shimony JS, Zhang D, Johnston JM, et al. Resting-state spontaneous fluctuations in brain activity: a new paradigm for presurgical planning using fMRI. Acad Radiol 2009; 16:578-83 CrossRef Medline

26. Tomasi D, Wang R, Wang GJ, et al. Functional connectivity and brain activation: a synergistic approach. Cereb Cortex 2014;24: 2619-29 CrossRef Medline

27. McHugo M, Rogers BP, Talati P, et al. Increased amplitude of low frequency fluctuations but normal hippocampal-default mode network connectivity in schizophrenia. Front Psychiatry 2015;6:92 CrossRef Medline

28. Fryer SL, Roach BJ, Ford JM, et al. Relating intrinsic low-frequency BOLD cortical oscillations to cognition in schizophrenia. Neuropsychopharmacology 2015;40:2705-14 CrossRef Medline

29. Yang M, Li J, Li Y, et al. Altered intrinsic regional activity and interregional functional connectivity in post-stroke aphasia. Sci Rep 2016;6:24803 CrossRef Medline

30. Zhu L, Fan Y, Zou Q, et al. Temporal reliability and lateralization of the resting-state language network. PLoS One 2014;9:e85880 CrossRef Medline

31. Yuan R, Di X, Kim EH, et al. Regional homogeneity of resting-state fMRI contributes to both neurovascular and task activation variations. Magn Reson Imaging 2013;31:1492-500 CrossRef Medline 